\title{
On Continuous Null Space Projections for Torque-Based, Hierarchical, Multi-Objective Manipulation
}

\author{
Alexander Dietrich, Alin Albu-Schäffer, and Gerd Hirzinger \\ Institute of Robotics and Mechatronics \\ German Aerospace Center (DLR), Germany \\ Contact: alexander.dietrichedlr.de
}

\begin{abstract}
The technological progress in the field of robotics results in more and more complex manipulators. However, having an increasing number of degrees of freedom raises the question of how to use them effectively. In turn, establishing manipulators in human environments, e.g., as service robots, calls for the fulfillment of various constraints and tasks at the same time. In the context of torque controlled robotic systems, we provide an approach to simultaneously deal with a multitude of tasks and constraints which are arranged in a hierarchy, utilizing the large number of actuated joints of the manipulator. To this end, we propose a continuous null space projection technique to consider unilateral constraints, singular Jacobian matrices and dynamic variations of the priority order within the hierarchical structure. We show that activating and deactivating tasks as well as crossing singularities does not lead to a discontinuous control law. Simulations and experiments on the humanoid Justin of the German Aerospace Center (DLR) validate our approach. The presented concept is supposed to contribute to whole-body control frameworks.
\end{abstract}

\section{INTRODUCTION}

Compared to industrial applications in which task execution is a low-dimensional issue, having robots in human environment calls for the fulfillment of various multi-dimensional tasks simultaneously. That comprises, for example, the interaction with unstructured environment and humans in the workspace of the manipulator. Systems like the humanoid Justin [1] are equipped with a large number of degrees of freedom (DOF) to be capable of meeting that criterion.

Being in need of executing several tasks at the same time naturally raises the question of a task hierarchy since in general it is not possible to realize all tasks simultaneously. Methods for such a prioritization have been thoroughly developed in the last decades. Most of them utilize null space projection techniques [2], [3] to provide a redundancy resolution. Based on that, sophisticated frameworks to incorporate a variety of different objectives have been designed [4], [5]. However, some open questions remain, e.g., how to deal with constraints within the hierarchy which are not active permanently. One example is reactive collision avoidance which only becomes relevant when an object is approaching the robot or a self-collision is close [6], [7], [8], see Fig. 1. Usually, one does not want to "lock" DOF just to reserve them for a (momentarily deactivated) task. In this context, various methods have been developed. In [9], the so-called dynamical systems approach is used to scale task contributions online. Self-collision avoidance movements are blended

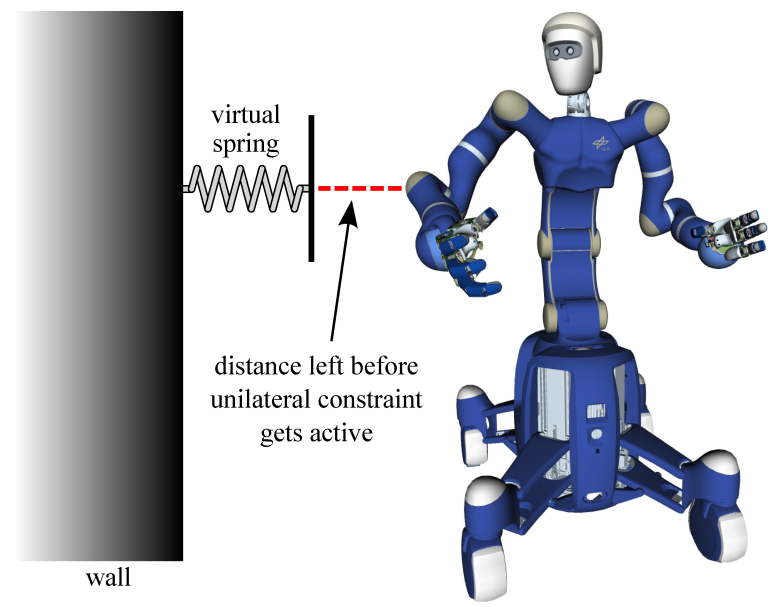

Fig. 1. Unilateral constraint (virtual spring) to avoid a collision with the wall.

with a whole-body motion control to change the priority order in [10]. Brock et al. propose a dynamic hierarchy [11]. Therein, obstacle avoidance is realized in the null space of a task. However, it is given a higher priority if that null space reveals not to be sufficient to avoid the collision. A suitable coefficient is calculated online to induce such a transition. Continuity is provided by smoothing the transition instead of modifying the control law in [12]. The framework acts on the kinematic level where joint velocities are the inputs to the robot. Another elaborate approach on the kinematic level has been proposed by Mansard et al. [13]. The paper introduces a new inversion operator to ensure continuity and applies it to a visual servoing scenario. In [14], an extension for a hierarchy of tasks and unilateral constraints is made. The extension to the dynamic level is provided in [15], but leads, however, to very complex formulations which are difficult to parametrize and use. Another method to deal with discontinuous inverses is to utilize damped least-squares techniques [16], leading to the question of the proper parameterization of the damping terms. These singularity-robust inverses (SRI) are widely used in the field of inverse kinematics. However, a simple and intuitive solution, which provides full control over the critical directions and slopes of the transition, is still missing.

This paper provides a framework for a dynamic hierarchy based on a new and very simple formulation for the null 
space projection computation. In contrast to most of the state of the art approaches, we do not rely on the kinematic but the dynamic level with joint torque interface. Our approach manages to deal with singular Jacobian matrices, dynamic hierarchies and unilateral constraints with respect to null space projector calculations. In the latter case, the mentioned undesired "locking" can be completely avoided. Our main contribution is the theoretical derivation and evaluation of a null space projection shaping technique which allows to selectively regulate the torque gradient during the transition process, independent of the singular values of the Jacobian matrix. We close the gap between the abstract mathematical structure of a task hierarchy and the directed influence on real physical values in the robotic system such as torques. Discontinuities can be "stretched" and distributed over a well-defined range to comply with any physical constraints. While controlling and fully specifying the exact transition behavior, we ensure that no errors from the ideal behavior occur outside this transient phase. The approach is based on a very intuitive interpretation of null space projections and poses no numerical problems when approaching singularities. We show that only the behavior in the critical directions is altered by the transition shaping while the other directions remain unaffected. We provide simulations and experimental results on the torque controlled robotic system Justin to show the performance of our redundancy resolution concept. Amongst others, the practically relevant application of selfcollision avoidance [6] has been chosen as an example. Our approach is equally applicable for a velocity interface in terms of well-directed limiting of joint accelerations. However, the kinematic case is beyond the scope of this paper and will be highlighted in a subsequent work. Here, we restrict to the force/torque interface.

The paper is organized as follows. First of all, we recall some basic knowledge about null space projection and point to the arising of discontinuities in Sec. II. In Sec. III, we derive our null space projection shaping equations. Simulations and experimental data are given in Sec. IV.

\section{FUNDAMENTALS AND PROBLEM STATEMENT}

A common way to induce a task hierarchy is to utilize null space projection techniques [2]. Given a robot with $n$ DOF and joint position vector $\boldsymbol{q} \in \mathbb{R}^{n}$, a primary task from priority level 1 (highest priority level) with dimension $m<n$ shall be described by a virtual constraint $\boldsymbol{f}(\boldsymbol{q})=\mathbf{0}$ to which a Jacobian matrix $\boldsymbol{J}=\frac{\partial \boldsymbol{f}(\boldsymbol{q})}{\partial \boldsymbol{q}} \in \mathbb{R}^{m \times n}$ belongs. Initially, $\boldsymbol{J}$ is supposed to be non-singular. The torques $\tau_{\text {sec }} \in \mathbb{R}^{n}$ from a lower priority level 2 (secondary task) may then be projected into its null space via the projection matrix

$$
\boldsymbol{N}=\boldsymbol{I}-\boldsymbol{J}^{T} \boldsymbol{J}^{+T}
$$

so that the commanded torque equals

$$
\boldsymbol{\tau}_{\mathrm{cmd}}=\boldsymbol{J}^{T} \boldsymbol{F}_{\text {prim }}+\boldsymbol{N} \boldsymbol{\tau}_{\mathrm{sec}} .
$$

The primary task force is represented by $\boldsymbol{F}_{\text {prim }} \in \mathbb{R}^{m}$. The matrix $\boldsymbol{J}^{+}$in (1) expresses the generalized inverse of $\boldsymbol{J}$. That operation is not unique and has to be further parameterized.
Inverting $\boldsymbol{J}$ requires the definition of a weighting matrix $\boldsymbol{W} \in \mathbb{R}^{n \times n}:$

$$
\boldsymbol{J}^{+, \boldsymbol{W}}=\boldsymbol{W}^{-1} \boldsymbol{J}^{T}\left(\boldsymbol{J} \boldsymbol{W}^{-1} \boldsymbol{J}^{T}\right)^{-1} .
$$

Frequently, $\boldsymbol{W}$ is set to the identity matrix $\boldsymbol{I}$. The resulting $\boldsymbol{J}^{+, \boldsymbol{I}}$ is then called the Moore-Penrose pseudoinverse ${ }^{1}$.

From a numerical point of view, the inversion is mostly done by applying a singular value decomposition (SVD):

$$
\boldsymbol{J}=\boldsymbol{U} \boldsymbol{S} \boldsymbol{V}^{T}
$$

with $\boldsymbol{U} \in \mathbb{R}^{m \times m}$ being a unitary matrix, $\boldsymbol{S} \in \mathbb{R}^{m \times n}$ a rectangular diagonal matrix containing the singular values $\sigma_{1}$ to $\sigma_{m}$ and $\boldsymbol{V} \in \mathbb{R}^{n \times n}$ a unitary matrix. Subsequently, the inversion of $\boldsymbol{J}$ by means of (3) with $\boldsymbol{W}=\boldsymbol{I}$ expressed in SVD components is ${ }^{2}$

$$
\begin{aligned}
\boldsymbol{J}^{+} & =\boldsymbol{J}^{T}\left(\boldsymbol{J} \boldsymbol{J}^{T}\right)^{-1} \\
& =\boldsymbol{V} \boldsymbol{S}^{T} \boldsymbol{U}^{T}\left(\boldsymbol{U} \boldsymbol{S} \boldsymbol{V}^{T} \boldsymbol{V} \boldsymbol{S}^{T} \boldsymbol{U}^{T}\right)^{-1} \\
& =\boldsymbol{V} \boldsymbol{S}^{T}\left(\boldsymbol{S} \boldsymbol{S}^{T}\right)^{-1} \boldsymbol{U}^{T} \\
& =\boldsymbol{V} \boldsymbol{S}^{+} \boldsymbol{U}^{T} .
\end{aligned}
$$

Commonly, the inversion of $\boldsymbol{S}$ in (6) is implemented by inverting the diagonal elements and cancelling the singular values for $\boldsymbol{S}^{+}$that are smaller than a specified tolerance $\varepsilon \in \mathbb{R}^{+}$. That operation can be interpreted as omitting the respective right-singular vectors in $\boldsymbol{V}$ or giving up the control of these directions. Recall that the columns in $\boldsymbol{V}$ form an orthonormal basis of $\mathbb{R}^{n}$. It shall be noted that cancelling the singular values less than $\varepsilon$ is an arbitrary choice to deal with the singularity while inverting the diagonal elements of $\boldsymbol{S}$. However, the occurence of discontinuities becomes evident at this point and is actually induced by this arbitrary choice. If the rank of the Jacobian matrix changes, threshold $\varepsilon$ is crossed, causing a discontinuous $\boldsymbol{S}^{+}$as the corresponding diagonal element jumps from $1 / \varepsilon$ to 0 . That effect propagates back to (1) and causes discontinuous projected torques from the lower levels.

Another alternative to cope with the inversion problem is to set a lower bound before inverting the singular values or to utilize damped least-squares techniques (SRI) [16], e.g.,

$$
\boldsymbol{J}^{*}=\boldsymbol{J}^{T}\left(\boldsymbol{J} \boldsymbol{J}^{T}+\lambda \boldsymbol{I}\right)^{-1} \text {. }
$$

Herein, superscript * indicates the damped inversion operation. A damping parameter $\lambda \in \mathbb{R}^{+}$can be specified which smoothes the transition. So far, various different approaches concerning damped least-squares methods have been proposed, e.g., to handle the problem of singularities in inverse kinematics. First solutions like choosing a constant damping factor [17] quickly revealed a crucial problem: Accuracy of the inverse away from the singularity and ensuring a smooth transition simultaneously is fairly unfeasible. Later designs based upon variable damping factors, e.g., dependent on the

\footnotetext{
${ }^{1}$ Alternatively, the so-called dynamically consistent pseudoinverse can be chosen using the inertia matrix $\boldsymbol{M}(\boldsymbol{q})$ such that $\boldsymbol{W}=\boldsymbol{M}(\boldsymbol{q})$. See [3] for a detailed discussion.

${ }^{2}$ As of now, the weighting matrix is omitted in the notation of the pseudoinverse as we set $\boldsymbol{W}=\boldsymbol{I}$.
} 
distance to the singularity [18] or its time derivative [19]. However, some problems remain. The choice of $\lambda$ is not intuitive and the direct consequence on physical values of the system is not defined as the treatment is done on an abstract mathematical level.

One always has to keep in mind that all projection-based methods are local redundancy resolutions and are not capable of providing a globally optimal performance [20].

\section{CONTINUOUS NULL SPACE PROJECTION SHAPING}

In this section, we will provide a new solution for a continuous null space projection which does not have the disadvantages of the existing techniques mentioned in the previous section. Our method allows to specifically limit the torque derivative during the transition phase. That closes the gap between the mathematical mechanisms of the projector calculation and the physical values of the real system. At first, let us take a closer look at the structure of the null space projector based on (1), (4) and (6).

\section{A. Intuitive Interpretation of the Null Space Projector}

Expressed in SVD components, (1) is described by

$$
\begin{aligned}
\boldsymbol{N} & =\boldsymbol{I}-\boldsymbol{V} \boldsymbol{S}^{T} \boldsymbol{U}^{T}\left(\boldsymbol{V} \boldsymbol{S}^{+} \boldsymbol{U}^{T}\right)^{T} \\
& =\boldsymbol{I}-\boldsymbol{V} \boldsymbol{S}^{T} \boldsymbol{U}^{T} \boldsymbol{U} \boldsymbol{S}^{+T} \boldsymbol{V}^{T} \\
& =\boldsymbol{I}-\boldsymbol{V} \underbrace{\boldsymbol{S}^{T} \boldsymbol{S}^{+T}}_{\boldsymbol{A}} \boldsymbol{V}^{T} .
\end{aligned}
$$

Herein

$$
\boldsymbol{A}=\operatorname{diag}\left(a_{1}, a_{2}, \ldots, a_{m}, \mathbf{0}_{1 \times(n-m)}\right)
$$

with

$$
a_{i}=\left\{\begin{array}{ll}
0 & \text { if } \sigma_{i}<\varepsilon \\
1 & \text { otherwise }
\end{array} \quad \forall 1 \leq i \leq m .\right.
$$

In (10) it becomes evident that only the right-singular vectors in $\boldsymbol{V}$ are relevant for the null space projector ${ }^{3}$, whereas matrix $\boldsymbol{U}$ and the absolute singular values in $\boldsymbol{S}$ do not have any influence on the result. The latter can be observed when considering the so-called activation matrix $\boldsymbol{A} \in \mathbb{R}^{n \times n}$. This matrix is supposed to contain either 1 (active) or 0 (inactive) diagonal elements. The $i$-th diagonal element refers to the $i$-th column vector in $\boldsymbol{V}$ and either activates that direction or locks it, when close to a singularity.

Since the singular values of the Jacobian matrix are not relevant, we need to consider only the directions of the constraint.

\section{B. Considering a 1 DOF System}

A $n=1$ DOF system is illustrated in Fig. 2. The depicted mass may move horizontally, its location is described by $z$. At $z=z_{\text {pot }}$, a repulsive, unilateral potential field begins in order to avoid a collision with the wall. The potential shall be the high priority task, whereas arbitrary tasks determine

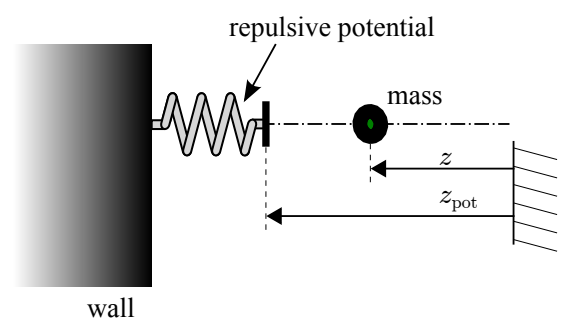

Fig. 2. 1 DOF system with primary task (repulsive potential) to avoid a collision of the mass with the wall.

the mass behavior in the null space of the collision avoidance task. The Jacobian matrix of the primary task is simply

$$
\boldsymbol{J}=\sigma \boldsymbol{J}_{1 \times 1}
$$

where the direction $\boldsymbol{J}_{1 \times 1}=[-1]$ is invariant and the singular value $\sigma$ is extracted from $\boldsymbol{J}$ beforehand. A SVD of (13) leads to (4) with $\boldsymbol{U}=[1], \boldsymbol{V}=[-1], \boldsymbol{S}=[\sigma]$. Due to the consideration of a 1 DOF system, simplifications in the calculation of the null space projector follow:

$$
N=1-S S^{+}=1-a_{1} .
$$

All matrices from (10) degenerate to scalars herein.

\section{Desired Transition Behavior for the 1 DOF System}

The discontinuity stated in (12) raises the question: Which behavior for the null space projector do we actually desire? Evidently, at least a continuous transition between 0 and 1 is required. Moreover, a desired behavior would be to specifically influence the transient behavior of values which are affected in the end: the projected torques or their derivatives, respectively. In this respect, we shape $N=N_{\text {des }}(z)$. The variable $z$ determines the state of the activation. An example for $z$ is a joint angle to describe the state of activation of a repulsive potential to avoid a mechanical end stop. Other examples are the distance between manipulator and an external object to describe the closeness to a collision (see Fig. 2) or the singular value $\sigma$ itself to express the distance to the singularity of the Jacobian matrix.

Following (14), the required activator is

$$
a_{1, \mathrm{des}}=1-N_{\mathrm{des}}(z) .
$$

A desired behavior could be to limit $\partial N_{\text {des }}(z) / \partial t$. Projecting a secondary task $\tau_{\mathrm{sec}}$ into the null space of the primary task, we obtain the control input

$$
\begin{aligned}
\tau_{\text {sec,proj }} & =N_{\text {des }}(z) \tau_{\mathrm{sec}} \\
\dot{\tau}_{\text {sec,proj }} & =\frac{\partial N_{\mathrm{des}}(z)}{\partial z} \frac{\partial z}{\partial t} \tau_{\mathrm{sec}}+N_{\mathrm{des}}(z) \dot{\tau}_{\mathrm{sec}} .
\end{aligned}
$$

Obviously, $N_{\text {des }}(z)$ must be at least of type $C^{1}$ in order to ensure continuity of (17). For the further analysis, we make the following assumptions:

\footnotetext{
${ }^{3}$ Actually, only the first $m$ column vectors in $\boldsymbol{V}$ are relevant here. Thus, a reduced SVD suffices.
} 
1) We neglect $\dot{\tau}_{\text {sec }}$. This is valid as we assume the transition to be faster than the changing of torque $\tau_{\mathrm{sec}}$ from a regular task.

2) A maximum or worst case $\tau_{\text {sec }}$ can be specified. If this is not possible, an online calculation or measurement is provided.

3) We are able to estimate a maximum or worst case value for $\partial z / \partial t$. If this is not possible, we are able to calculate or measure $\partial z / \partial t$ online.

Concerning $N_{\text {des }}(z)$, we suggest a piecewise defined function containing a third order polynomial, i.e.,

$$
\begin{gathered}
N_{\text {des }}(z)= \begin{cases}1 & \text { if } z<z_{1} \\
g(z) & \text { if } z_{1} \leq z \leq z_{2} \\
0 & \text { otherwise }\end{cases} \\
g(z)=c_{1} z^{3}+c_{2} z^{2}+c_{3} z+c_{4}
\end{gathered}
$$

with $\left[z_{1}, z_{2}\right]$ defining the interval from unconstrained null space projection to full locking. Limiting the term $N_{\max }^{\prime}=\max \left(\left|\partial N_{\operatorname{des}}(z) / \partial z\right|\right)$ allows to "stretch" the torque change/discontinuity over a well-defined range. More precisely, a maximum torque derivative $\dot{\tau}_{\text {sec,proj,max }}$ can be specified $^{4}$ :

$$
N_{\max }^{\prime}=\dot{\tau}_{\text {sec,proj,max }}\left|\frac{\partial z}{\partial t} \tau_{\text {sec }}\right|^{-1} .
$$

One choice for $\dot{\tau}_{\text {sec,proj,max }}$ is a parameterization according to the performance of the torque controller. We set the following conditions for (19): $g\left(z_{1}\right)=1, g^{\prime}\left(z_{1}\right)=0, g\left(z_{2}\right)=$ $0, g^{\prime}\left(z_{2}\right)=0, \max \left(\left|g^{\prime}(z)\right|\right)=-g^{\prime}\left(\frac{z_{2}+z_{1}}{2}\right)=N_{\max }^{\prime}$. That over-determined system of equations can be solved by adding the range $\left\{z_{2}-z_{1}\right\}$ to the set of unknown parameters $\left\{c_{1}, c_{2}, c_{3}, c_{4}\right\}$ from (19).

Notice that limiting $N_{\max }^{\prime}$ for (19) is a conservative

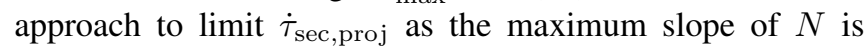
only reached once within the transition interval. It will be shown in the following simulation that using a third order polynomial is only marginally more conservative than the fastest continuous transition, i.e., an affine function, but it leads to a smoother behavior.

Fig. 3 shows results for constant values of $N_{\max }^{\prime}=$ 2 , 5, 20. The upper plots depict the activator. At $z=$ $z_{\text {pot }}=0.8$, the primary task is fully activated, the respective Jacobian matrix has full rank, and the DOF is locked for all lower priority tasks than the highest one. The parameterization $N_{\max }^{\prime}$ can be identified in the bottom plots when regarding the maximum slope of $N$.

As described above, other transition functions than (19) may be used. Applying an affine relation would bring the benefit of a constant $\partial N(z) / \partial z$ within the transition phase instead of the quadratic ones shown in Fig. 3 (bottom). It can be shown generally that the interval size would reduce to $2 / 3$ of the original size. However, a lack of smoothness would result at the beginning of the interval and at $z=0.8$.

\footnotetext{
${ }^{4}$ If $\partial z / \partial t$ or $\tau_{\mathrm{sec}}$ are taken from measurements or estimations online, a loop is closed. In order to ensure passivity, a separate proof has to be made.
}
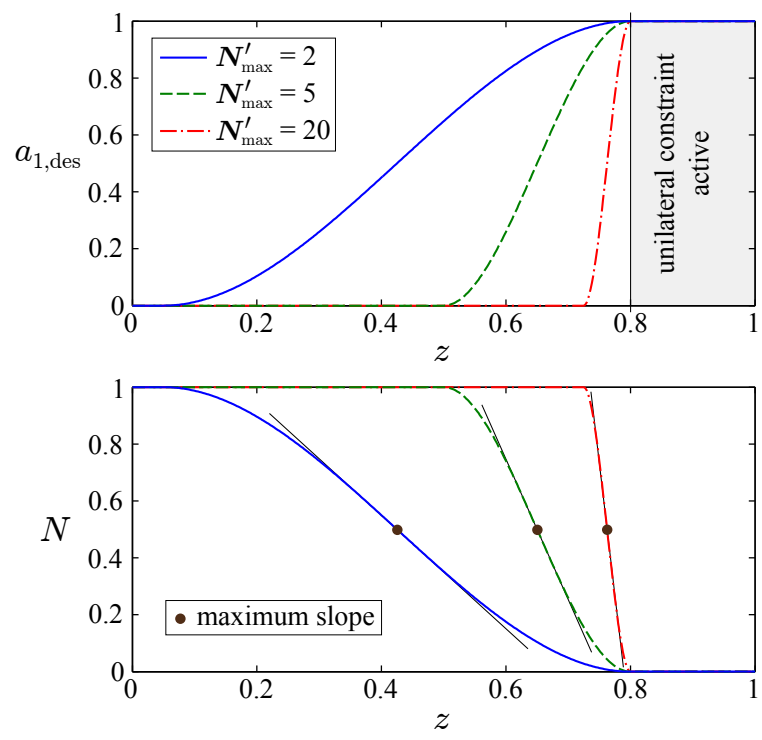

Fig. 3. Examples for transition shaping in the case of a 1 DOF system (normalized/dimensionless coordinate $z$ ).

\section{Considering $a(1 \times n)$ Constraint}

The 1 DOF case is trivial as the null space projector equals a complete fade-out of the secondary task torque at the activation point of the constraint. Now, we will extend the mechanism to the nontrivial $(1 \times n)$ case such that $\boldsymbol{J}=\sigma \boldsymbol{J}_{1 \times n} \in \mathbb{R}^{1 \times n}$. Hence, $\boldsymbol{J}_{1 \times n}$ is already normalized. Starting from (10), we apply the desired diagonal activator matrix $\boldsymbol{A}_{\text {des }}$ and obtain

$$
\begin{aligned}
\boldsymbol{N} & =\boldsymbol{I}-\boldsymbol{V} \boldsymbol{A}_{\mathrm{des}} \boldsymbol{V}^{T} \\
& =\boldsymbol{I}-\boldsymbol{J}_{1 \times n}^{T} a_{1, \mathrm{des}} \boldsymbol{J}_{1 \times n} .
\end{aligned}
$$

Herein, only the first element $a_{1 \text {,des }}$ of $\boldsymbol{A}_{\mathrm{des}}$ is relevant. Due to the SVD of row vector $\boldsymbol{J}$, the first column of the $\boldsymbol{V}$-matrix yields $\boldsymbol{v}_{1}=\boldsymbol{J}_{1 \times n}^{T}$. Knowing the general relation of (22) brings along a major advantage: The SVD becomes unnecessary. The null space projector is obtained by pure multiplications.

Unilateral constraints of multi-DOF manipulators are typically of this kind.

\section{E. The General $(m \times n)$ Case}

We now consider the Jacobian matrix $\boldsymbol{J}=\boldsymbol{J}_{m \times n} \in \mathbb{R}^{m \times n}$ for the general case $m \leq n$. This case is relevant if a general Jacobian matrix $\boldsymbol{J}_{m \times n}$ becomes singular. The arising singularity can be dealt with by choosing $\boldsymbol{A}_{\mathrm{des}}=\boldsymbol{A}_{\mathrm{des}}(\boldsymbol{\sigma})$ such that the singular values $\boldsymbol{\sigma} \in \mathbb{R}^{m}$ are consulted.

The question arises how to design $a_{1 \text {,des }}$ to $a_{m \text {,des. }}$ To interpret (10) and (11) in this case, we introduce the projection $\boldsymbol{P}$ of $\boldsymbol{N}$ into the directions of $\boldsymbol{V}$. Recall that the columns of $\boldsymbol{V}$ span an orthonormal basis.

$$
\begin{aligned}
\boldsymbol{P}_{i} & =\boldsymbol{v}_{i}^{T} \boldsymbol{N} \\
& =\boldsymbol{v}_{i}^{T}-\boldsymbol{v}_{i}^{T} \boldsymbol{V} \boldsymbol{A}_{\mathrm{des}} \boldsymbol{V}^{T} \\
& =\underbrace{\left(1-a_{i, \mathrm{des}}\right)}_{\text {see }(14)} \boldsymbol{v}_{i}^{T} \forall 1 \leq i \leq m .
\end{aligned}
$$


We observe that (23) is similar to (14) but projected into the direction $\boldsymbol{v}_{i}$. Therefore, shaping of the transition function in the $(m \times n)$ case has to be regarded as a multidimensional design of the "stretching" in the first $m$ directions $\boldsymbol{v}_{1}$ to $\boldsymbol{v}_{m}$. Of course, the projections into the remaining $n-m$ directions are not affected by the transition:

$$
\boldsymbol{P}_{j}=\boldsymbol{v}_{j}^{T} \boldsymbol{N}=\boldsymbol{v}_{j}^{T} \forall m<j \leq n .
$$

That invariance of the remaining directions is one main benefit of the approach compared to most of the scaling and blending techniques wherein secondary tasks may be completely disabled when higher priority ones become active [9], or the priority order is never ensured completely [21].

\section{F. Desired Transition Behavior in the General $(m \times n)$ Case}

Nevertheless, the question of the design of $\boldsymbol{A}_{\text {des }}$ in the $(m \times n)$ case is still open. One approach to handle the complexity is to decompose the lower level torques, to consider the contributions in the critical directions, and to apply the methods from Sec. III-C. Notice that an online decomposition and a feedback into the generation process of $\boldsymbol{A}_{\mathrm{des}}$ closes an additional loop. However, as stated in assumption 1), the transition is supposed to be significantly faster than the changing of torques from the lower levels. Thus, the effect is expected to be quite limited. An offline consideration is more conservative but does not close a further loop. The simplest, still quite effective solution would be to choose constant transition behaviors for all $m$ directions. At this point it shall be noted that the design in the $(1 \times n)$ case is straightforward when applying such an online decomposition. As $m=1$, the simplification

$$
\boldsymbol{P}_{1}=\left(1-a_{1, \mathrm{des}}\right) \boldsymbol{J}_{1 \times n}
$$

follows due to the fact that $\boldsymbol{v}_{1}^{T}=\boldsymbol{J}_{1 \times n}$. Therefore, the lower level torques are supposed to be projected just into the primary task direction $\boldsymbol{J}_{1 \times n}$.

\section{SIMULATIONS AND EXPERIMENTS ON UNILATERAL CONSTRAINTS}

In this section, we provide some simulations and experimental results to validate the proposed method for unilateral constraints. Mostly in literature, reactive collision avoidance is not handled as a primary task exactly due to the described discontinuity problem, but rather as a low level task. That does not correspond to real demands of the applications. The proposed framework can optimally handle this issue which will be shown in the experiments on the real robotic system at the end of Sec. IV.

\section{A. Simulations on the 3 DOF System}

We performed simulations for a planar system as depicted in Fig. 4 (left). It consists of three links and three revolute joints. Viscous joint friction is modeled and the masses are decoupled. As task with low priority, a Cartesian impedance is chosen whose goal it is to lead the tool center point (TCP) to the goal configuration. The primary task is defined by a singularity avoidance which is designed via a

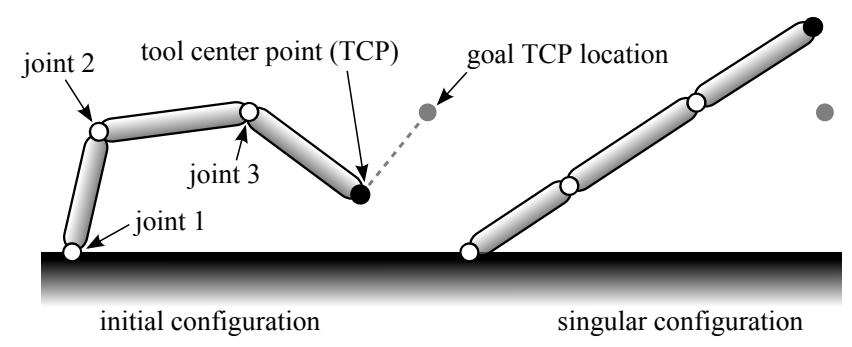

Fig. 4. Schematic structure of the 3 DOF system.
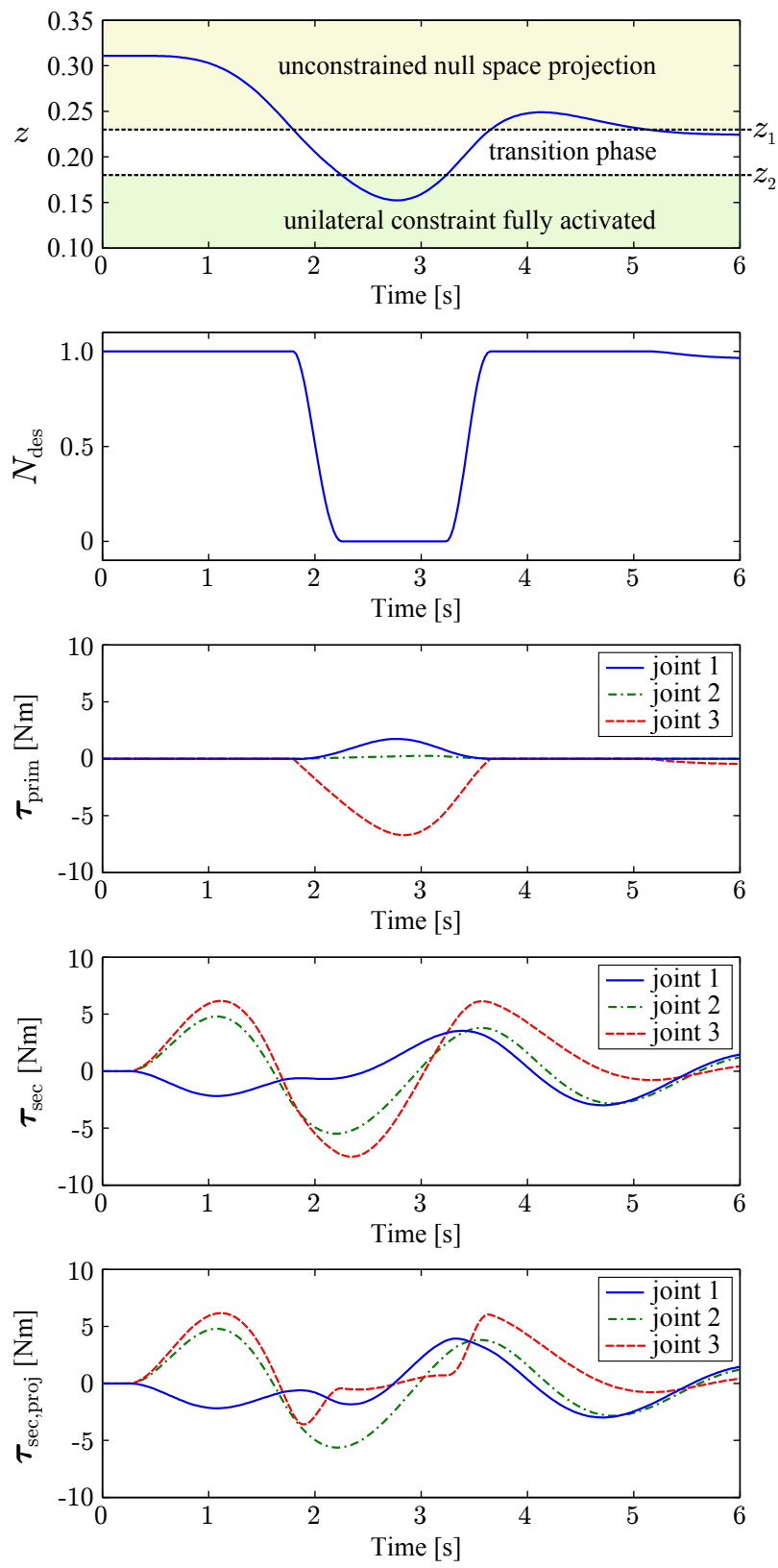

Fig. 5. Activation of a unilateral constraint during a 3 DOF system simulation. The transition is specified by $N_{\max }^{\prime}=30$. ( $z$ : manipulability measure of $\boldsymbol{J}_{\mathrm{sec}} ; \boldsymbol{\tau}_{\mathrm{prim}}$ : singularity avoidance torques; $\boldsymbol{\tau}_{\mathrm{sec}}$ : Cartesian impedance torques). 


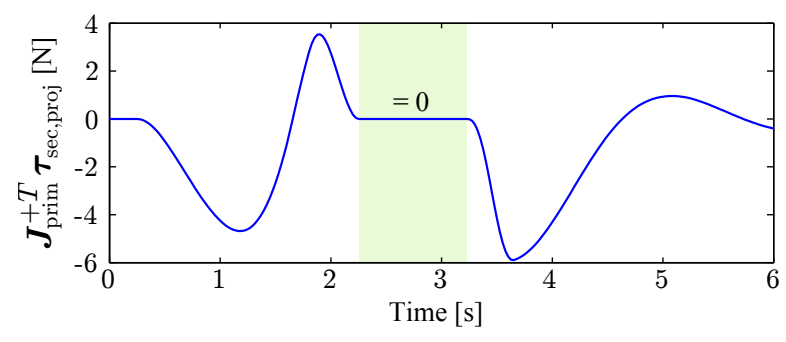

Fig. 6. Contribution of the projected lower priority task torques in the constraint direction (continuous null space). While the constraint is fully activated, no secondary torque passes.

repulsive potential based on the kinematic manipulability measure [22] of the Jacobian matrix $\boldsymbol{J}_{\mathrm{sec}}$ related to the Cartesian impedance. That avoidance with torque command $\tau_{\text {prim }}$ is a unilateral constraint which gets activated if the manipulability measure falls below a specified value. The Cartesian reference trajectory of the TCP (Fig. 4 left) is designed such that the singularity indicated in Fig. 4 (right) is approached. A conflict between the tasks is provoked. Recall that the singular configuration could never be reached by the Cartesian impedance in a steady state. The primary task would outplay the Cartesian impedance finally. Fig. 5 depicts the results for the first $6 \mathrm{~s}$ of the simulation. Within this time interval, a full transition occurs which will be analyzed in the following.

Starting from the initial configuration (Fig. 4 left), the end effector moves towards the singular configuration (right). The primary task gets activated at $t=1.8 \mathrm{~s}$ for the first time. The transition with $N_{\max }^{\prime}=30$ can be observed in the upper plot of Fig. 5 with $z$ expressing the manipulability measure. The second plot shows $N_{\text {des }}(t)$. In this experiment, we designed the primary task (third diagram) to start from $z_{1}$ on. The bottom diagrams show the Cartesian impedance torques $\boldsymbol{\tau}_{\text {sec }}$ and their projections $\tau_{\text {sec,proj }}$ into the null space of the primary task. We want to draw attention to the projected torques when the primary task becomes activated. As the singularity avoidance mainly requires intervention at the third joint $(1.8 \mathrm{~s}<t<3.6 \mathrm{~s})$, the respective projected impedance torque alters the most.

When the unilateral constraint is fully activated, no projection of the Cartesian impedance torques may remain in the primary task direction. Therefore, we multiply $\boldsymbol{\tau}_{\text {sec,proj }}$ by $\boldsymbol{J}_{\text {prim }}^{+T}$. Fig. 6 shows the results. Within the time interval of full activation (shaded rectangle), no torque comes through. Hence, the condition of an undisturbed priority order is met. The control inputs $\boldsymbol{\tau}_{\text {cmd }}=\tau_{\text {prim }}+\tau_{\text {sec,proj }}$ are depicted in Fig. 7 (top). In contrast, a discontinuous null space projection (bottom) based on a common matrix inversion leads to significant discontinuities in the torques. These can be observed at time $1.8 \mathrm{~s}, 3.6 \mathrm{~s}$ and $5.4 \mathrm{~s}$.

In this simulation, a steady state in the continuous case is reached after $6 \mathrm{~s}$ asymptotically. In that final configuration, $z$ is a little lower than $z_{1}$ as indicated in Fig. 5 (upper plot). No further full transition occurs after $6 \mathrm{~s}$ since the intervention $\forall 1.8 \mathrm{~s}<t<3.6 \mathrm{~s}$ induced a null space or
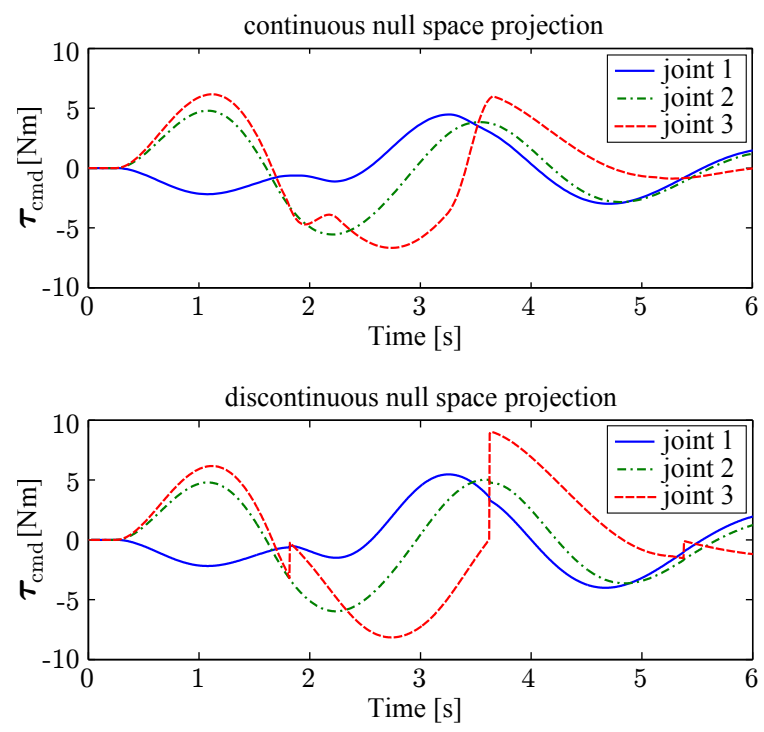

Fig. 7. Control inputs for different null space projection methods Significant discontinuities in the case of a common null space projection can be identified.

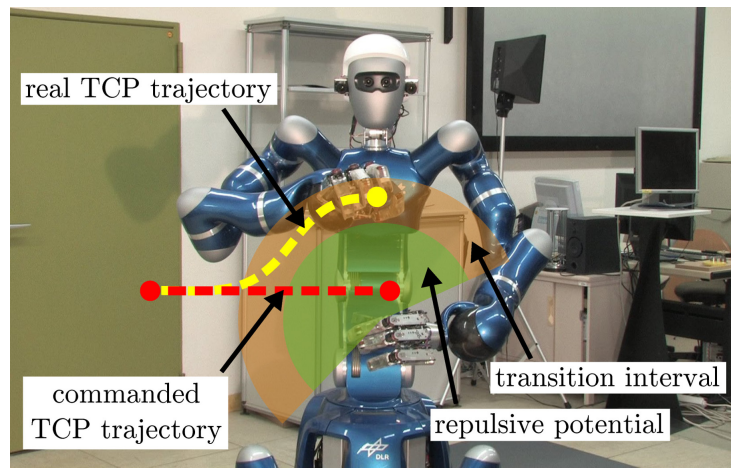

Fig. 9. The robot avoids a self-collision between the hands. The snapshot is taken in the intermediate configuration at $t=4 \mathrm{~s}$.

internal motion that reconfigured the manipulator to comply with the singularity avoidance constraint.

\section{B. Experiments on the Humanoid Justin}

We performed experiments on Justin to verify our algorithm. In the following, we use a 6 DOF Cartesian impedance of the right tool center point (TCP) as the secondary task. The commanded trajectory of the TCP is illustrated by the red, dashed line in Fig. 9. The maximum translational velocity is set to $0.5 \mathrm{~m} / \mathrm{s}$. The primary task is a repulsive potential to achieve self-collision avoidance between left and right hand. The respective algorithm for that avoidance application is explained in detail in [6]. The repulsive potential is depicted by the green sector in Fig. 9. The transition interval is illustrated by the adjacent corridor. Fig. 8 shows the results of the experiment. The activation/deactivation of the primary task can be observed in the upper left plot. The corresponding measured joint torques $\boldsymbol{\tau}_{\text {meas }}$ (left, bottom) do not indicate any discontinuities during the transition phase. As the goal TCP configuration is set to be infeasible, torques from the Cartesian impedance remain in the intermediate 

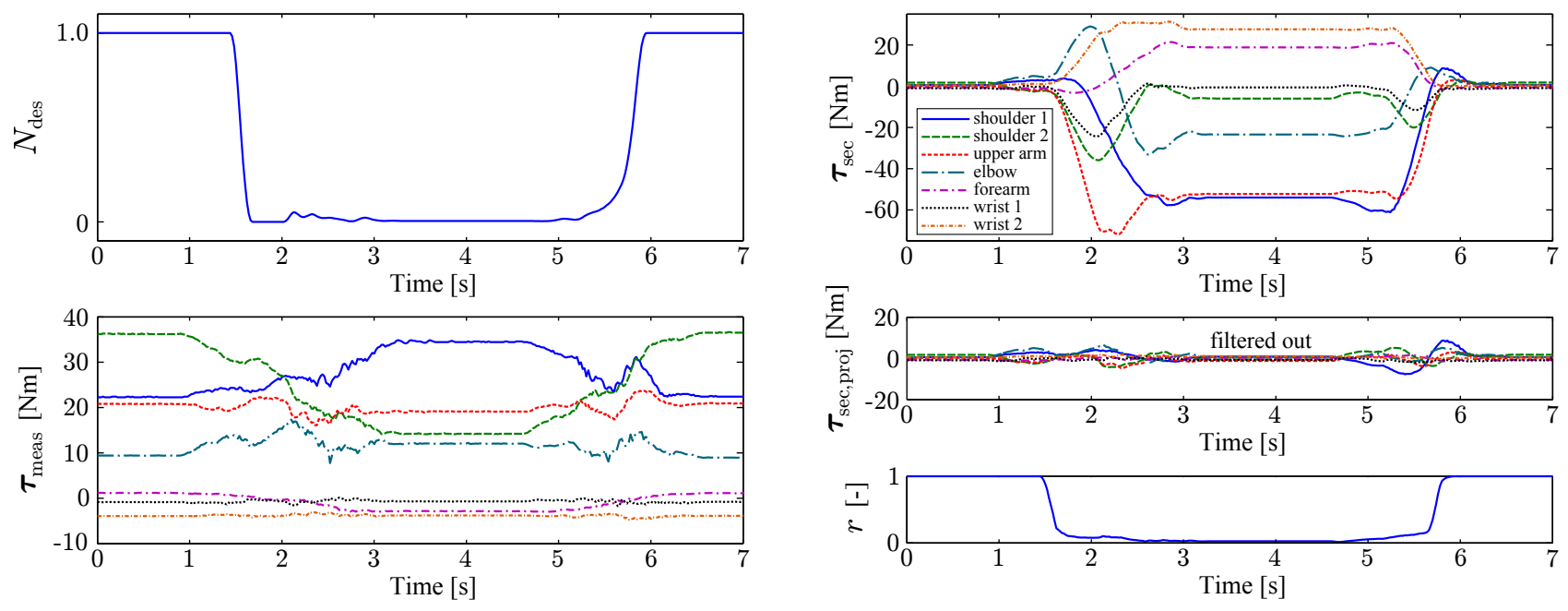

Fig. 8. Primary task is a unilateral constraint (self-collision avoidance potential field), a Cartesian impedance serves as secondary task. The torque plots refer to the 7 right arm joint torques. The legend for the distinction of all torque curves is given in the upper right diagram. The joints are defined as follows: shoulder 1 (roll), shoulder 2 (pitch), upper arm (roll), elbow (pitch), forearm (roll), wrist 1 (pitch), wrist 2 (pitch).

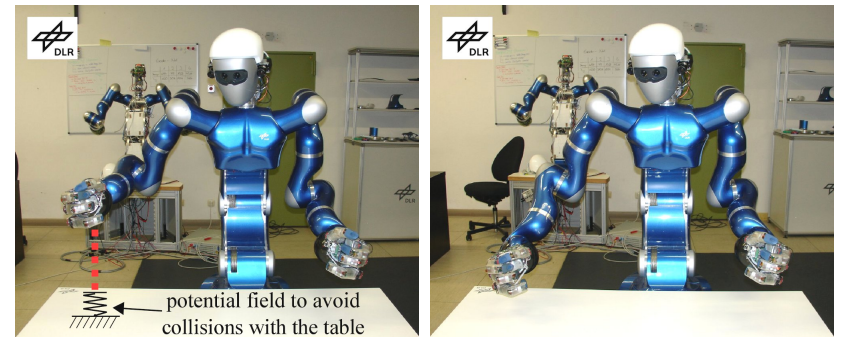

Fig. 10. The robot avoids a collision with the table.

configuration which is shown in Fig. 9. However, they are largely filtered out by the null space projection as it can be seen in the right diagrams of Fig. 8. The bottom plot on the right shows the ratio $r=\left\|\boldsymbol{\tau}_{\text {sec,proj }}\right\| /\left\|\boldsymbol{\tau}_{\text {sec }}\right\|$ to indicate the feasibility of the secondary task. Notice that the direction of the primary task changes permanently during the experiment corresponding to the locations of both hands.

In the second experiment we also use the 6 DOF Cartesian impedance of the right tool center point (TCP) as the secondary task. The continuous trajectory of the TCP describes a motion of $0.3 \mathrm{~m}$ downward along the vertical axis. After $0.25 \mathrm{~m}$, the primary task gets activated which is defined by a unilateral constraint, i.e., a repulsive potential to avoid a collision with the table, see Fig. 10. In the left diagrams of Fig. 11, the behavior for the shaped null space projection is shown. The upper plot depicts $N_{\text {des }}$, whereas the lower plots show torques measured at selected joints. Except for some measurement noise, the signals appear smooth. The right diagrams show the results for a common null space projection based on singular value cancellation as described in (12). Obviously, the two tasks compete at the discrete activation border. Particularly between $7 \mathrm{~s}$ and $8 \mathrm{~s}$, several transitions are provoked which lead to the respective peaks in the torque measurements. Amplitudes up to $50 \mathrm{Nm}$ can be identified. Notice that the lower plots display the real torques that appear at the joints. The commanded torques attain values of almost $90 \mathrm{Nm}$ but they are not feasible due to the high frequency of the transitions.

The supplementary video shows some exemplary scenes demonstrating the null space projection shaping. That includes comparisons between continuous and discontinuous null spaces in simulation as well as an experiment on Justin.

\section{CONCLUSIONS}

Multi-DOF robotic systems require a proper redundancy resolution to handle the numerousness of degrees of freedom. If multiple tasks are to be executed simultaneously, a hierarchy between them has to be introduced. In this paper, we provided the framework for such a prioritization in the case of torque control. We considered unilateral constraints and singular Jacobian matrices within the hierarchy and proposed an approach that did not lead to a discontinuous control law. We selectively shaped the transition in the respective direction while the other directions remained unaffected. Moreover, we had full control over the duration and shape of the transition, avoiding the exceeding of any physical limitation.

Simulations and experimental results on the humanoid Justin of the German Aerospace Center (DLR) validated our method for three different kinds of unilateral constraints, i.e., singularity avoidance, self-collision avoidance and collision avoidance with external objects.

Future works will focus on the design of a unified framework to allow multi-objective, dynamic mobile manipulation based on our recent work [23]. Therein, the issue of discontinuous null space projections has not been treated yet. In this context, the dynamic hierarchy approach proposed here will play an important role. Moreover, we will formulate the proposed approach for velocity controlled systems.

\section{REFERENCES}

[1] C. Borst, T. Wimböck, F. Schmidt, M. Fuchs, B. Brunner, F. Zacharias, P. R. Giordano, R. Konietschke, W. Sepp, S. Fuchs, C. Rink, A. Albu- 

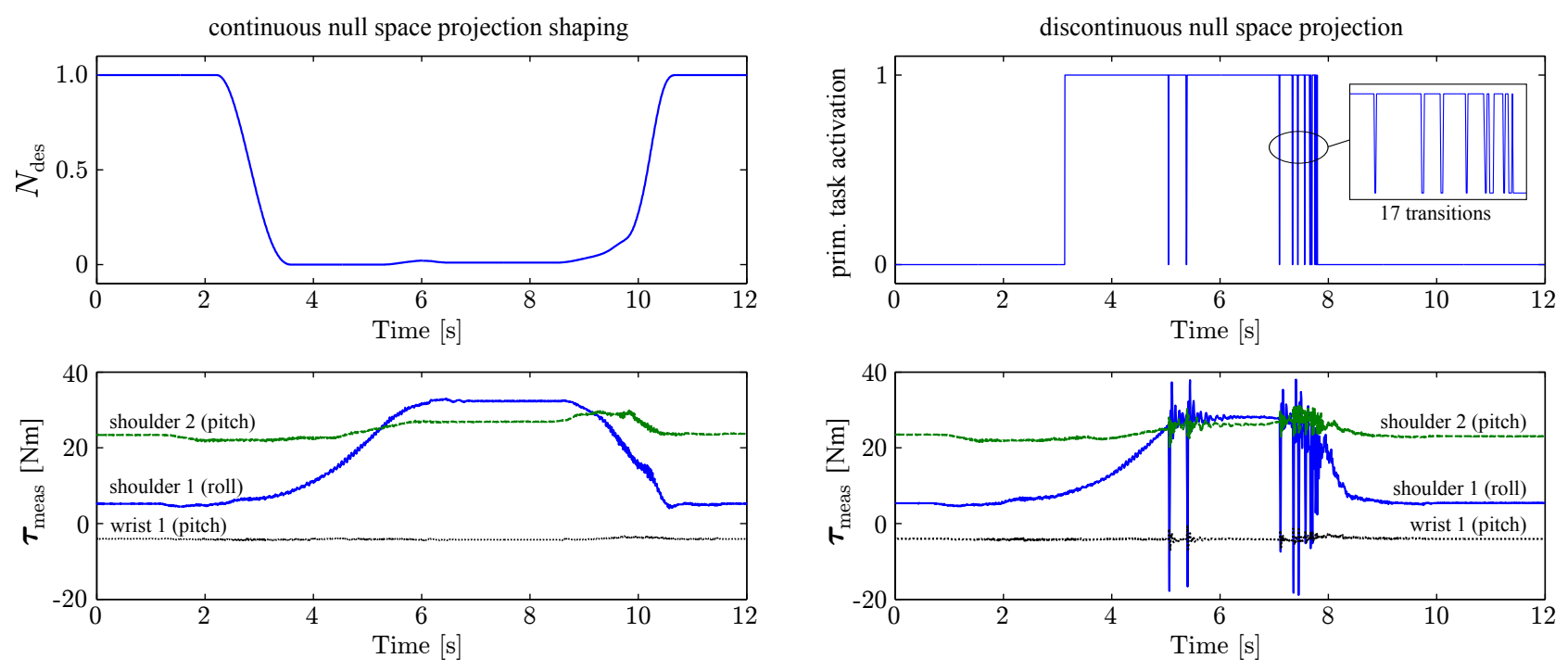

Fig. 11. Null space projections implemented on the humanoid Justin. The primary task is a unilateral constraint (collision avoidance), a Cartesian impedance serves as the secondary task.

Schäffer, and G. Hirzinger, "Rollin' Justin - Mobile Platform with Variable Base," in Proc. of the 2009 IEEE International Conference on Robotics and Automation, May 2009, pp. 1597-1598.

[2] B. Siciliano and J.-J. Slotine, "A General Framework for Managing Multiple Tasks in Highly Redundant Robotic Systems," in Proc. of the 5th International Conference on Advanced Robotics, June 1991, pp. 1211-1216.

[3] O. Khatib, "A Unified Approach for Motion and Force Control of Robot Manipulators: The Operational Space Formulation," IEEE Journal of Robotics and Automation, vol. RA-3, no. 1, pp. 43-53, February 1987.

[4] L. Sentis and O. Khatib, "Synthesis of Whole-Body Behaviors through Hierarchical Control of Behavioral Primitives," International Journal of Humanoid Robotics, vol. 2, no. 4, pp. 505-518, January 2005.

[5] O. Khatib, L. Sentis, J. Park, and J. Warren, "Whole-Body Dynamic Behavior and Control of Human-like Robots," International Journal of Humanoid Robots, vol. 1, no. 1, pp. 29-43, March 2004.

[6] A. Dietrich, T. Wimböck, H. Täubig, A. Albu-Schäffer, and G. Hirzinger, "Extensions to Reactive Self-Collision Avoidance for Torque and Position Controlled Humanoids," in Proc. of the 2011 IEEE International Conference on Robotics and Automation, May 2011, pp. 3455-3462.

[7] H. Sugiura, M. Gienger, H. Janssen, and C. Goerick, "Reactive Self Collision Avoidance with Dynamic Task Prioritization for Humanoid Robots," International Journal of Humanoid Robots, vol. 7, no. 1, pp. 31-54, 2010.

[8] O. Stasse, A. Escande, N. Mansard, S. Miossec, P. Evrard, and A. Kheddar, "Real-Time (Self)-Collision Avoidance Task on a HRP-2 Humanoid Robot," in Proc. of the 2008 IEEE International Conference on Robotics and Automation, May 2008, pp. 3200-3205.

[9] L.-P. Ellekilde and H. I. Christensen, "Control of Mobile Manipulator using the Dynamical Systems Approach," in Proc. of the 2009 IEEE International Conference on Robotics and Automation, May 2009, pp. 1370-1376.

[10] H. Sugiura, M. Gienger, H. Janssen, and C. Goerick, "Real-Time Collision Avoidance with Whole Body Motion Control for Humanoid Robots," in Proc. of the 2007 IEEE/RSJ International Conference on Intelligent Robots and Systems, October 2007, pp. 2053-2058.

[11] O. Brock, O. Khatib, and S. Viji, "Task-Consistent Obstacle Avoidance and Motion Behavior for Mobile Manipulation," in Proc. of the 2002 IEEE International Conference on Robotics and Automation, May 2002, pp. 388-393.
[12] J. Lee, N. Mansard, and J. Park, "Intermediate Desired Value Approach for Continuous Transition among Multiple Tasks of Robots," in Proc. of the 2011 IEEE International Conference on Robotics and Automation, May 2011, pp. 1276-1282.

[13] N. Mansard, A. Remazeilles, and F. Chaumette, "Continuity of Varying-Feature-Set Control Laws," IEEE Transactions on Automatic Control, vol. 54, no. 11, pp. 2493-2505, November 2009.

[14] N. Mansard, O. Khatib, and A. Kheddar, "A Unified Approach to Integrate Unilateral Constraints in the Stack of Tasks," IEEE Transactions on Robotics, vol. 25, no. 3, pp. 670-685, June 2009.

[15] N. Mansard and O. Khatib, "Continuous Control Law from Unilateral Constraints: Application to Reactive Obstacle Avoidance in Operational Space," in Proc. of the 2008 IEEE International Conference on Robotics and Automation, May 2008, pp. 3359-3364.

[16] A. Deo and I. Walker, "Overview of Damped Least-Squares Methods for Inverse Kinematics of Robot Manipulators," Journal of Intelligent Robotic Systems, vol. 14, no. 1, pp. 43-68, September 1995.

[17] C. W. Wampler, "Manipulator Inverse Kinematic Solutions Based on Vector Formulations and Damped Least-Squares Methods," IEEE Transactions on Systems, Man, and Cybernetics, vol. 16, no. 1, pp. 93-101, January 1986.

[18] Y. Nakamura and H. Hanafusa, "Inverse Kinematic Solutions With Singularity Robustness for Robot Manipulator Control," Journal of Dynamic Systems, Measurement, and Control, vol. 108, no. 3, pp. 163-171, September 1986.

[19] L. Kelmar and P. Khosla, "Automatic Generation of Kinematics for a Reconfigurable Modular Manipulator System," in Proc. of the 1988 IEEE International Conference on Robotics and Automation, April 1988, pp. 663-668.

[20] D. R. Baker and C. W. Wampler, "On the Inverse Kinematics of Redundant Manipulators," International Journal of Robotics Research, vol. 7, no. 2, pp. 3-21, April 1988.

[21] J. Salini, V. Padois, and P. Bidaud, "Synthesis of Complex Humanoid Whole-Body Behaviors: a Focus on Sequencing and Tasks Transitions," in Proc. of the 2011 IEEE International Conference on Robotics and Automation, May 2011, pp. 1283-1290.

[22] C. Ott, Cartesian Impedance Control of Redundant and FlexibleJoint Robots, ser. Springer Tracts in Advanced Robotics. Springer Publishing Company, Berlin Heidelberg, 2008, vol. 49.

[23] A. Dietrich, T. Wimböck, and A. Albu-Schäffer, "Dynamic WholeBody Mobile Manipulation with a Torque Controlled Humanoid Robot via Impedance Control Laws," in Proc. of the 2011 IEEE/RSJ International Conference on Intelligent Robots and Systems, September 2011 , pp. 3199-3206. 